\title{
Agua, poder y hegemonía entre actores estatales y no estatales en Turquía, Siria e Iraq
}

\author{
Water, Power and Hegemony \\ between State and Non-state \\ Actors in Turkey, Syria and Iraq
}

\section{GILBERTO CONDE*}

Resumen: Podría parecer que las negociaciones para regular el reparto de aguas de ríos internacionales contribuyen a la paz. En un tenor diferente, el presente estudio coincide con diversos autores que han encontrado que las negociaciones y los acuerdos delimitan y a la vez legitiman las relaciones asimétricas de poder y los conflictos en las cuencas internacionales. Esto se ha verificado en la cuenca del Tigris y el Éufrates en las relaciones por el agua entre Turquía, Siria, Iraq y sus poblaciones kurdas. En el artículo se reflexiona sobre la cuestión de la hidrohegemonía y se trata de enriquecer el concepto. política.

Palabras clave: Tigris; Éufrates; kurdos; ISIs; geopolítica; hidro-

Abtract: It would seem that negotiations to regulate the sharing of water of international rivers contribute to peace. At variance with such a view, this article coincides with authors that have found that negotiations and agreements define and legitimize asymmetrical power relations and conflict in international basins. This has been found to occur in the Tigris and Euphrates basin in the water re-

Recepción: 23 de junio de 2016. / Aceptación: 10 de agosto de 2016.

* El Colegio de México, A.C., gilberto.conde@colmex.mx 
lations between Turkey, Syria, Iraq and their Kurdish populations. The article proposes a reflection on hydrohegemony, while seeking to enrich the concept.

Key words: Tigris; Euphrates; Kurds; ISIs; Geopolitics; Hydropolitics.

\section{Introducción}

En la historia de las negociaciones por el agua del Tigris y el Éufrates, Turquía ha utilizado sus ventajas para ejercer una suerte de hidrohegemonía, cuando no hidrodominación, sobre los kurdos de su país y sobre los Estados vecinos. Durante el siglo XX, se establecieron varios acuerdos que, a pesar de ser temporales, regulan la distribución del recurso en la cuenca. Entre 2000 y 2011, ocurrieron cambios en las relaciones entre los tres Estados, lo que llevó a nuevos acuerdos. ¿Se estaba transitando hacia una integración internacional en la cuenca gracias a los acuerdos por el agua? ¿O se tendía a fijar los términos de la hidrohegemonía turca con base en los nuevos acuerdos y el nuevo mecanismo de regulación con ganancias relativas para diferentes Estados? La situación inaugurada con la represión y las guerras en Siria en 2011 llevó a que cambiara de nuevo la situación y quedaran al descubierto las fragilidades de los acuerdos de inicios del siglo y de la esperanza del establecimiento de un régimen internacional.

Aunque se hace una breve referencia a las evoluciones anteriores, en este artículo se discute principalmente el periodo inaugurado con el inicio de los años 2000. Se argumenta que el conflicto por el agua y las relaciones de poder no desaparecieron durante los años de cordialidad y construcción de interdependencias. Esto indica, como se ha discutido en un artículo anterior, que los conflictos por el agua rara vez son exclusivamente por el líquido y que suelen reflejar los conflictos o la colaboración que se dan entre los distintos actores en otros ámbitos. ${ }^{1}$

${ }^{1}$ Gilberto Conde, "El agua entre Turquía, Siria e Iraq, ¿ ¿barómetro de conflictos?”, Regions E Cohesion, vol. 4, núm. 2, verano de 2014, pp. 81-100. 
En el primer apartado, se recapitula rápidamente la discusión teórica acerca del agua como lubricante de la paz o como elemento de conflicto. En el segundo, se recapitula cómo se desarrollaron las relaciones por el agua durante el siglo xx en la cuenca que nos convoca. Lo conciso de este análisis se debe tanto a problemas de espacio como a que estos puntos se analizarán con mayor profundidad en otra publicación. En el tercero, se exploran los acontecimientos ocurridos durante la primera década del siglo xxI, y, en el cuarto, se reflexiona acerca de los cambios acaecidos durante el lustro iniciado con las rebeliones populares árabes de 2011, particularmente la de Siria. Incluye la toma de presas por la organización Estado Islámico (que ha tenido varios nombres y se conoce por varios de ellos, como Estado Islámico de Iraq y Levante o las siglas de esto en árabe -Daesh-, en inglés -ISIS, ISIL- o en español y francés, EIIL). A lo largo del texto, se considera también el papel de los kurdos, tanto los que participan en el movimiento kurdo de liberación como los del partido dominante en el Gobierno Regional del Kurdistán iraquí.

\section{La hidrohegemonía}

Después de que durante la segunda mitad de la década de 1990 y posteriormente varios académicos que trabajaban sobre el agua en el Medio Oriente insistieron en que el recurso - más que motivo de luchas de poder, como se venía aseverando- podía servir para facilitar los procesos de paz, ${ }^{2}$ un grupo de estudiosos de las relaciones internacionales por recursos hídricos basado

${ }^{2}$ Véanse, por ejemplo, Masahiro Murakami, Managing Water for Peace in the Middle East: Alternative Strategies, Tokio, United Nations University Press, 1995; J. Anthony Allan (ed.), Water, Peace and the Middle East: Negotiating Resources in the Jordan Basin, Londres, St. Martin's Press, 1996; Hussein Amery y Aaron T. Wolf, "Water, Geography, and Peace in the Middle East: An Introduction", en Hussein Amery y Aaron T. Wolf (eds.), Water in the Middle East: A Geography of Peace, Austin, Texas University Press, 2000, pp. 1-18; Aaron T. Wolf, “'Hydrostrategic Territory' in the Jordan Basin: Water, War, and Arab-Israeli Peace Negotiations”, en Amery y Wolf (eds.), Water in the Middle East, op. cit., pp. 63-120; Hillel I. Shuval y Hassan Dwiek (eds.), Water Resources in the Middle East: The Israeli-Palestinian Water Issues: From Conflict to Cooperation, Berlín, Springer, 2007. 


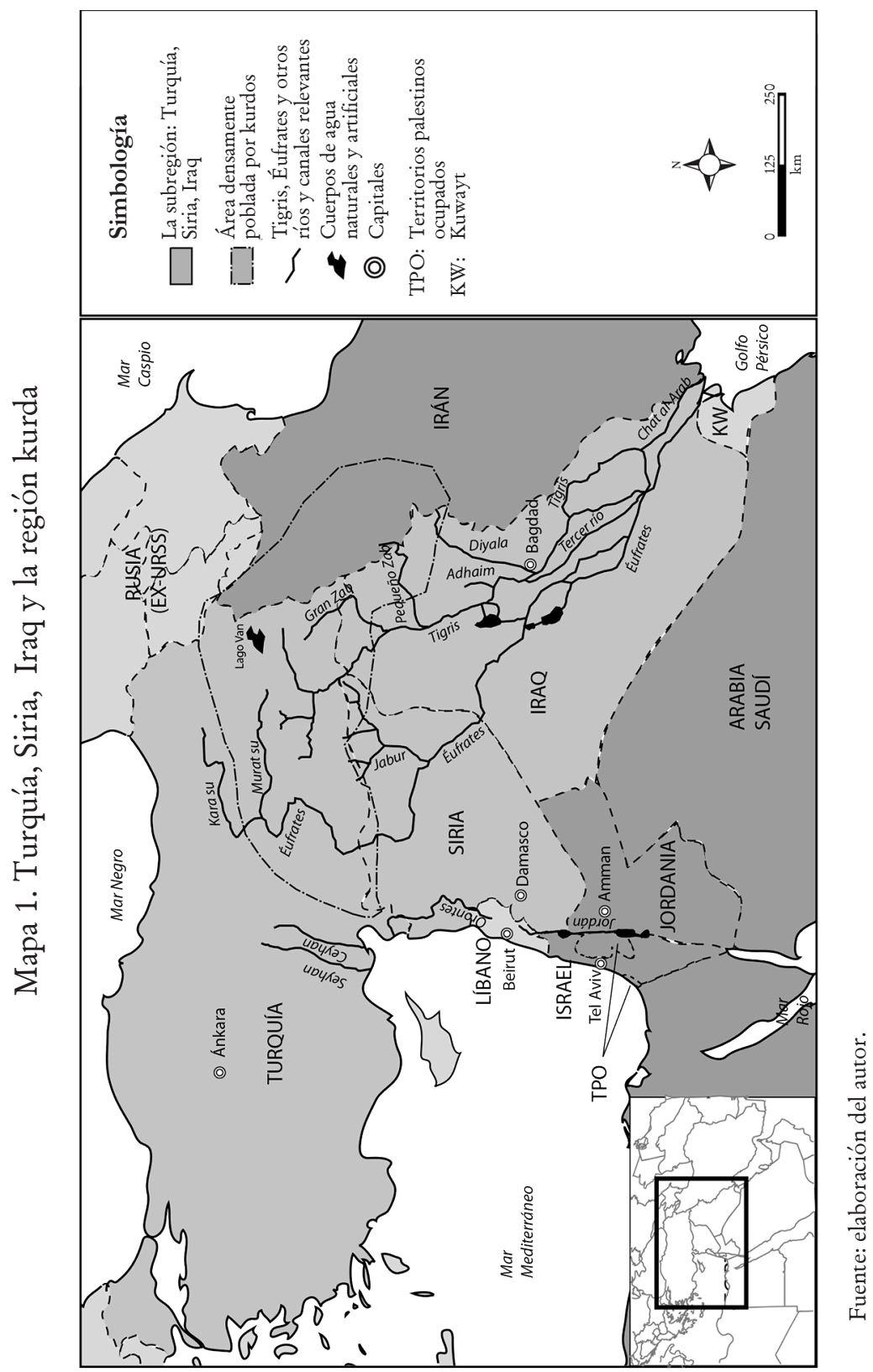


en Londres desarrolló durante la década siguiente el concepto de la hidrohegemonía. ${ }^{3}$ Explicaron de manera convincente que los conflictos por el agua no resultan de una falsa disyuntiva entre guerra y cooperación. Por el contrario, el conflicto por el agua persiste, aunque haya negociaciones que lleven a acuerdos e incluso a cooperación. Las relaciones hídricas también son relaciones de poder. Como el conflicto es una realidad, es necesario advertir su intensidad, y las implicaciones de la cooperación.

Por supuesto, la hidrohegemonía no expresa únicamente agresividad y coerción o intentos de dominación directa, aunque también los ha habido. Es a lo que Zeitoun et al. llaman "poder duro", 4 y que Gramsci llamaría simplemente "dominación". ${ }^{5}$ Sin embargo, esto que podríamos llamar "hidrodominación” es una forma de control menos eficaz que la derivada de lo que Zeitoun et al. llamaron "poder blando", o, si se desea emplear el vocabulario gramsciano, la "hegemonía”. En ésta, el grupo dominante logra que el subalterno acepte su condición de inferioridad por medios distintos a la coerción o la violencia. Zeitoun et al. concluyen que el poderoso logra que los otros deseen lo mismo que él mediante numerosos instrumentos de poder blando, "desde pagos colaterales y corrupción hasta la persuasión y la promoción del deseo a emular el éxito". Esta forma de poder incluye lo que consideran un aspecto positivo de atracción, pero también uno negativo, de repulsión de ciertas agendas y temas para conservar el statu quo asimétrico.

Así, tanto la negociación como la cooperación por el agua entre Estados no sólo incluyen sino que reifican las relaciones

${ }^{3}$ Entre otros, Mark Zeitoun y Jeroen Warner, "Hydro-Hegemony: A Framework for Analysis of Trans-Boundary Water Conflicts", Water Policy, vol. 8, 2006, pp. 435-460; Jeroen Warner, "Hydro-Hegemonic Politics: A Crossroads on the Euphrates-Tigris?”, en Kai Wegerich y Jeroen Warner (eds.), The Politics of Water: A Survey, Londres, Routledge, 2010, pp. 119-141; Mark Zeitoun, Power and Water in the Middle East: The Hidden Politics of the Palestinian-Israeli Water Conflict, Londres, I. B. Tauris, 2011.

${ }^{4}$ Mark Zeitoun, Naho Mirumachi y Jeroen Warner, "Transboundary Water Interaction II: The Influence of 'Soft' Power”, International Environmental Agreements: Politics, Law and Economics, vol. 11, núm. 2, 2011, pp. 159-178.

${ }^{5}$ Antonio Gramsci, Selections from the Prison Notebooks of Antonio Gramsci, eds. y trads. Quintin Hoare y Geoffrey Nowell-Smith, Nueva York, International Publishers, 1971. 
de hidrohegemonía. ${ }^{6}$ A las formas ya mencionadas de afirmación del poder blando se pueden agregar las propuestas y las negociaciones de simulación (token), que buscan aparentar una voluntad de negociación o la realización de acuerdos provisionales que mantengan la amenaza de volver a políticas más agresivas, de poder duro, o incluso la firma de acuerdos definitivos, que suelen canonizar la relación inicua.

¿Cuál es el papel del agua en los conflictos enmascarados por la negociación entre Estados? ¿Realmente desea o necesita más agua el hidrohegemón? En términos estrictamente económicos, el agua, aunque importante para un Estado poderoso, dominado por una clase capitalista fuerte, podría ser de un interés relativamente menor en comparación con un Estado y una burguesía más dependientes de la agricultura. ${ }^{7}$ Éste podría ser el caso de Turquía, Israel o, en otra región del mundo, Estados Unidos en relación con países con los que comparten cuencas. No obstante, el agua puede ser útil para una serie de objetivos muy diversos, incluidos los económicos, pero también los de política interior y exterior o incluso los simbólicos. Las publicaciones sobre la política del agua están llenas de ejemplos al respecto.

\section{Las relaciones por el agua en el Tigris y el Éufrates en el siglo $\mathrm{xx}$}

Desde la segunda mitad del siglo xx, Turquía ha sido el Estado más poderoso económica, militar y diplomáticamente de las cuencas del Tigris y el Éufrates. El agua de estos ríos le ha servido para fines económicos, sociales y políticos. Le ha servido para generar energía hidroeléctrica, promover las industrias de la construcción y de las finanzas, comercializar equipamiento de irrigación y fortalecer la agroindustria. En términos sociales, le ha servido para crear empleos y colonizar la parte de cuenca

${ }^{6}$ Jeroen Warner, Mark Zeitoun y Naho Mirumachi, “How 'Soft Power' Shapes Transboundary Water Interaction”, GWF Discussion Paper 1323, Canberra, Global Water Forum, junio de 2013.

7 Jan Selby, "The Geopolitics of Water in The Middle East: Fantasies and Realities”, Third World Quarterly, vol. 26, núm. 2, junio de 2005, pp. 329-349. 


\section{Mapa 2. Cuenca del Tigris y el Éufrates}

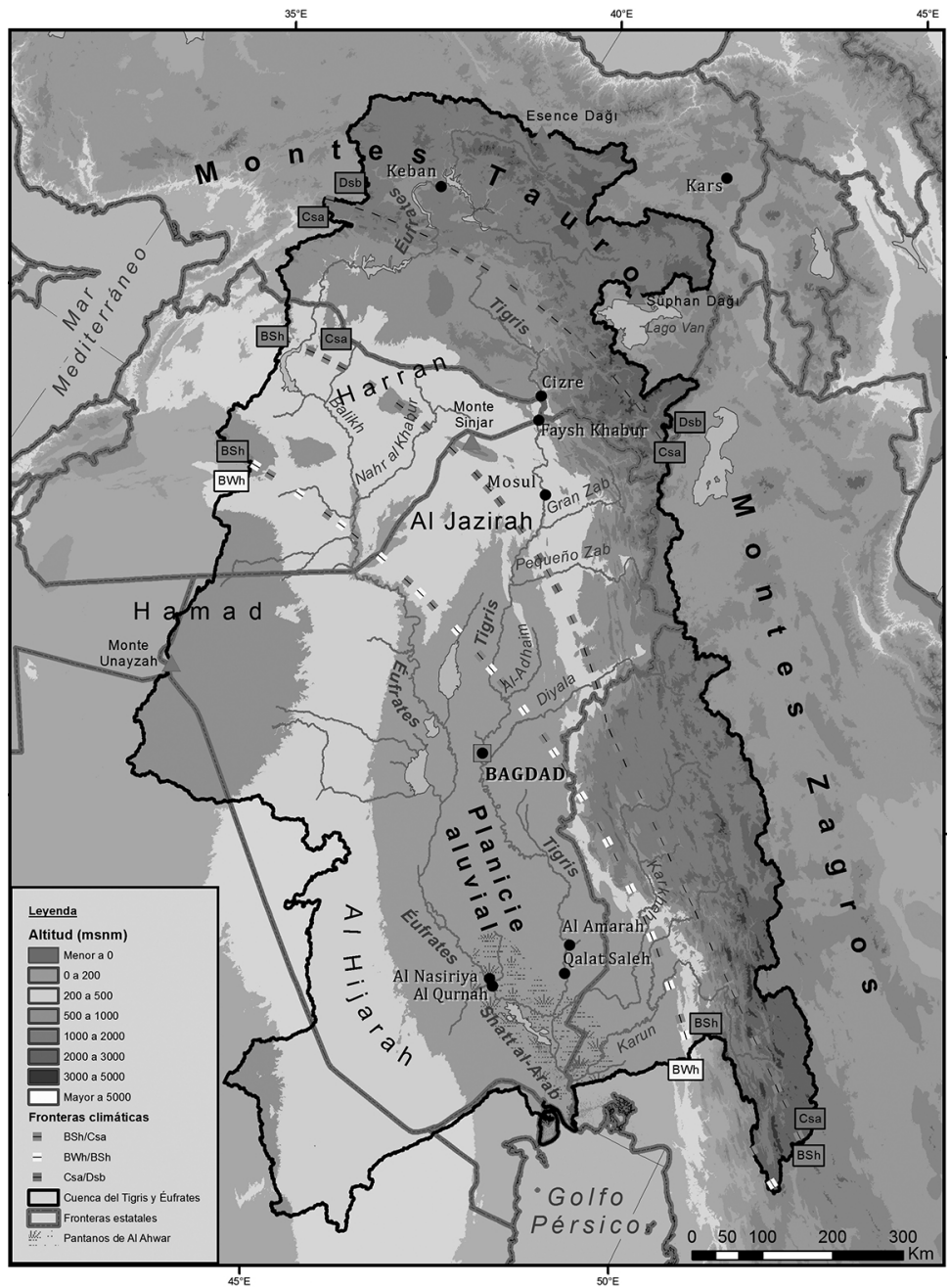

Proyección: Cónica Equidistante de Albers. Autor: Sebastián Estremo Paredes. 
que se encuentra dentro de su territorio. En términos de política interna, su utilidad ha sido enorme en la guerra contra el Partido de los Trabajadores del Kurdistán (PKK, por su nombre en kurdo) por medio del control de poblaciones, pero también de presión sobre otros Estados de la cuenca para obtener su colaboración en la lucha contra la guerrilla kurda. ${ }^{8}$ Asimismo, en el ámbito interno, no hay que desdeñar el aspecto simbólico del agua; los megaproyectos y la defensa de la soberanía turca sobre las aguas del Tigris y el Éufrates les han permitido a las autoridades de Ankara atizar el nacionalismo y con ello aumentar la hegemonía sobre su propia población. En política (y economía) exterior, los conflictos con negociaciones y amenazas le han servido al Estado turco para alcanzar una posición de poder respecto de sus vecinos sudorientales, Siria e Iraq.

Entre la década de 1920 y el final de la Segunda Guerra Mundial, Turquía no era la principal potencia en la cuenca, ya que, tras la desintegración del Imperio otomano después de la Primera Guerra Mundial, Siria e Iraq quedaron bajo mandatos francés y británico respectivamente. Los ciclos de negociaciones por el agua durante esa época reflejaban el poderío de las potencias europeas establecidas en la región. Franceses y británicos negociaron entre sí que éstos serían consultados en caso de que aquéllos quisieran edificar obras en los cursos de agua compartidos. Luego, ambos trataron con las autoridades de Turquía para llegar a acuerdos específicos. Francia consiguió un compromiso turco de entregar agua del Quwayq a la ciudad siria de Alepo. ${ }^{9}$ Pasada la Segunda Guerra Mundial, Siria obtuvo su independencia y Turquía dejó de honrar los acuerdos. Para la década de 1960, el consumo del lado turco ya había desecado el río. ${ }^{10}$ En 1939, Francia cedió la provincia siria de Alejandreta a Turquía, que renombró Hatay, y se comprometió a compartir las aguas del Orontes, que fluye desde Monte Líbano, pasando por el fértil valle del Ghab en Siria.

${ }^{8}$ Habib Ayeb, L'eau au Proche-Orient: la guerre n'aura pas lieu, París, Karthala, 1998; Gilberto Conde, "Kurdish Counter-hegemony in the Tigris and Euphrates Water Basins: Local Impacts Beyond Water Distribution in Turkey", Local Environment: The International Journal of Justice and Sustainability, 2016 (en prensa).

9 Tarek Majzoub, Les fleuves du Moyen-Orient, París, L'Harmattan, 1994. 2011.

${ }^{10}$ Wolfgang Wagner, Groundwater in the Arab Middle East, Heidelberg, Springer, 
Antes de que Turquía o Siria se interesaran por desarrollar seriamente la irrigación en la cuenca del Tigris y el Éufrates, hubo algunas negociaciones que parecían prometedoras. Era ya la época en la que en Estados Unidos la economía crecía promovida por las grandes obras de infraestructura, y la Tennessee Valley Authority conquistaba al mundo con su concepción integral del desarrollo hídrico de la cuenca. Iraq, ya independiente, aunque fuera en términos sólo formales, acordó apoyar la construcción de presas en Turquía con el fin de limitar las inundaciones en la baja Mesopotamia. Ankara, por su lado, se comprometió a consultar al gobierno de Bagdad antes de erigir obras hidráulicas río arriba. Ambos aceptaban armonizar sus proyectos. Al final, el acuerdo se convirtió en letra muerta. ${ }^{11}$

En las décadas siguientes, ya con Estados independientes, Turquía buscó afirmar su hegemonía, ya fuera en términos de dominación o de liderazgo, sobre los países ribereños inferiores, Siria e Iraq, a pesar de que, por lo menos algunos gobernantes iraquíes buscaron establecer la visión de cuenca para el manejo integral de los recursos. En los albores de lo que se conoció como la guerra fría árabe, ${ }^{12}$ en 1955, el gobierno de Turquía prácticamente vetó un crédito que el Banco Internacional para la Reconstrucción y el Fomento (BIRF, el actual Banco Mundial) le iba a dar a Siria para construir una presa en el Éufrates, y pocos años después consiguió el crédito para construir su propia presa en el río en el sitio de Keban, a pesar de que el mismo banco había solicitado con anterioridad que los tres países se pusieran de acuerdo para la construcción de infraestructura hidráulica en la cuenca del río si deseaban contar con su apoyo. ${ }^{13}$ Para escapar a las presiones turcas y de

${ }^{11}$ Majzoub, Lesfleuves du Moyen-Orient, op. cit.; Yüksel Inan, "Legal Dimensions of International Watercourse (Euphrates and Tigris)", en Ali Ihsan Bağış (ed.), Water as an Element of Cooperation and Development in the Middle East, Estambul, AynaHacettepe Universitesi-Friedrich Neuman Foundation, 1994, pp. 223-237.

${ }_{12}$ Malcolm H. Kerr, The Arab Cold War: Gamal Abd al-Nasir and his Rivals, 1958-1970, Londres, Oxford University Press, 1971.

${ }^{13}$ Raj Krishna, "International Watercourses: World Bank Experience and Policy", en J. Anthony Allan y Chibli Mallat (eds.), Water in the Middle East: Legal, Political and Commercial Implications, Londres, I. B. Tauris, 1995, pp. 29-54. 
sus aliados occidentales, Siria recurrió a préstamos soviéticos para construir su presa sobre el Éufrates. ${ }^{14}$

Hubo varios llamados a la cooperación hídrica en la cuenca, incluida una iniciativa iraquí a inicios de la década de 1960 que cayó en oídos sordos, ${ }^{15}$ y reuniones de una Comisión Técnica Mixta bi y tripartita desde mediados del decenio que no logró ni siquiera ponerse de acuerdo sobre el alcance de sus trabajos. ${ }^{16}$ Siguió una política de hechos consumados por cada uno de los actores estatales durante el resto del periodo.

Durante las décadas siguientes, las autoridades de Turquía intentaron utilizar su poder económico, político y militar, aprovechando su relación estratégica con Estados Unidos, para imponer sus decisiones en la cuenca del Tigris y el Éufrates y para, posteriormente, intentar obligar a Damasco y luego a Iraq para que reprimieran a los kurdos del PKK que desde sus territorios luchaban contra el régimen turco.

Entre 1973 y 1974, las autoridades turcas y sirias empezaron a llenar las grandes presas que habían construido sobre el Éufrates, y dejaron a Iraq con un flujo muy mermado de agua en la corriente. Las autoridades de Bagdad, sin embargo, decidieron acusar únicamente a las de Siria por la catástrofe hídrica que les aquejaba, ${ }^{17}$ y amenazaron con declararle la guerra. La mediación saudí y soviética evitó el choque y permitió la firma de un acuerdo de repartimiento de aguas, en el que Siria podía conservar $40 \%$ del torrente e Iraq, $60 \%$. El músculo militar de Bagdad le permitió conseguir un acuerdo benéfico. El poderío diplomático de Ankara le permitió seguir actuando como si sus presas se erigieran en otra parte del mundo.

Turquía siguió aprovechando sus ventajas estratégicas para, en la década siguiente, iniciar la construcción de otras dos grandes presas y anunciar el faraónico Proyecto del Sureste de Anatolia (GAP, por su nombre en turco), que incluía la edificación de 22 grandes presas, sin mencionar otras medias y pequeñas, con

${ }^{14}$ Thomas Naff y Ruth C. Matson, Water in the Middle East: Conflict or Cooperation?, Boulder, Westview Press, 1984.

${ }_{15}^{15}$ Majzoub, Les fleuves du Moyen-Orient, op. cit.

${ }^{16}$ Ayşegül Kibaroğlu, Building a Regime for the Waters of the Euphrates-Tigris River Basin, Londres, Kluwer Law International, 2002.

${ }^{17}$ Conde, "Kurdish Counter-hegemony...", op. cit. 
la finalidad de irrigar unas 1.7 millones de hectáreas. ${ }^{18} \mathrm{La}$ naturaleza hidrohegemónica del GAP, sin embargo, no sólo se traducía en el aprovechamiento de un volumen enorme de agua en comparación con el que podían tomar los proyectos de Siria y de Iraq.

También se expresaba en las presiones que ejercían los políticos de Ankara sobre los de Siria para que contribuyeran a desmantelar la guerrilla kurda que había iniciado la lucha armada en 1984 estableciendo bases de entrenamiento en Líbano. Entre 1987 y 1993 , se sucedieron varios acuerdos o intentos de acuerdo bilaterales en los que Turquía ofrecía agua a cambio de la colaboración total de Siria con los proyectos de seguridad turcos contra los kurdos. Fue así que en 1987 se estableció un acuerdo provisional mediante el cual Turquía le garantizaba un flujo de $500 \mathrm{~m}^{3} / \mathrm{s}$ de agua a Siria sobre el Éufrates. El acuerdo se ha convertido en norma permanente, aun en los mejores momentos de la relación bilateral.

No obstante, a inicios de 1990, Turquía redujo sorpresivamente el flujo del Éufrates durante un mes. En lugar de lograr la hidrodominación de Siria para que colaborara más estrechamente con la política antiinsurgente de Ankara, el presidente sirio Hafez al-Asad se puso de acuerdo con su archienemigo, el hombre fuerte iraquí, Saddam Husayn, para firmar un acuerdo bilateral para repartir las aguas que recibieran de río arriba en el Éufrates a una taza de $48 \%$ y $52 \%$ respectivamente. ${ }^{19}$ Este acuerdo, que modificaba para beneficio de Damasco el de 1974, delata el relativo fortalecimiento hidrohegemónico de Siria.

Desde 1993 hasta la amenaza turca de invadir Siria en 1999, el expediente del agua se mantuvo alto en las declaraciones y en los foros internacionales, como en Naciones Unidas a lo largo de las negociaciones de una Convención internacional de aguas para fines distintos a la navegación, pero fue prácticamente abandonado de las relaciones bilaterales o trilaterales en la cuenca.

${ }^{18}$ John F. Kolars y William A. Mitchell, The Euphrates River and the South East Anatolia Development Project, Carbondale-Edwardsville, Southern Illinois University Press, 1991.

${ }^{19}$ Greg Shapland, Rivers of Discord: International Water Disputes in the Middle East, Nueva York, St. Martin's Press, 1997. 


\section{La hidrohegemonía suave durante los años de entendimiento}

Con el nuevo siglo, mejoraron las relaciones entre los Estados de la cuenca del Tigris y el Éufrates, particularmente las que tienen que ver con el agua de los ríos. Como se ha argumentado, esto no significa que haya desaparecido la hidrohegemonía en las relaciones entre los tres Estados y menos aún respecto de los kurdos. Por el contrario, la forma en la que se dieron las relaciones por el agua confirma la tesis de que la negociación y la cooperación han representado la continuación del conflicto por otras vías.

Varios factores permitieron el mejoramiento de las relaciones, particularmente entre Turquía y Siria, aunque también entre ambos e Iraq, tanto antes como después de la intervención estadounidense. Destaca, entre éstos, que Damasco haya echado de su territorio al dirigente del rebelde PKK, Abdullah Öcalan, lo que permitió que fuera capturado cuando bajaba de un avión en África y entregado a las autoridades turcas, y que las autoridades sirias coincidieran con las de Turquía en cuanto al temor a que se fortaleciera la región autónoma del Kurdistán iraquí. Bashar al-Asad, al llegar a la presidencia de su país en 2000, empezó a buscar aliados comerciales a escala internacional y realizó gestos hacia Turquía. El triunfo electoral del islamista Partido de la Justicia y el Desarrollo (AKP, por su nombre en turco) en 2002 tuvo un peso significativo para mejorar la relación, ya que uno de los puntos importantes que les interesaban a sus electores era el mejoramiento de relaciones con otros países islámicos, particularmente con Siria. ${ }^{20}$

Así, durante la primera década del siglo Xxi, las administraciones de Turquía y Siria alcanzaron acuerdos comerciales, militares y culturales, con lo que mejoraron sus relaciones a un grado sin precedentes. Esto facilitó la relación bilateral respecto del agua. Durante el periodo, se perfiló una especie de hegemonía de las élites de Turquía sobre sus vecinos del sur en asuntos comerciales e industriales. Ciertos empresarios sirios esperaban

${ }^{20}$ Gilberto Conde, “Turquía e Iraq en las cambiantes relaciones internacionales de Siria”, en Luis Mesa Delmonte (coord.), Las relaciones exteriores de Siria, México, El Colegio de México, A.C., 2013, pp. 159-205. 
ver crecer sus capitales al convertirse en socios menores de sus símiles turcos.

En el tema del agua ocurrió algo análogo. Representantes de ambos países firmaron un Comunicado Conjunto el 23 de agosto de 2001 que ratificaron con el Documento de Implementación del 19 de junio del año siguiente. Los instrumentos indicaban que iban a coordinar ciertas actividades, pero el asunto de los repartos quedó sin definir y no se incluyó a Iraq, el país ribereño inferior. ${ }^{21}$ Turquía se comprometía a dejar pasar un volumen del fluido superior a lo acordado en 1987 y, en tiempos de sequía, Siria aceptaría un flujo menor al establecido. Las autoridades de Iraq bajo la ocupación estadounidense (2003-2011) se quejaban esporádicamente y denunciaban la exclusión de su país de la negociación y de los documentos firmados, aunque con poco éxito.

Da la impresión de que el acuerdo incluyó un entendimiento, probablemente tácito, por medio del cual los dirigentes sirios silenciaban el tema del acuerdo tripartita definitivo de aguas al que había aspirado junto con Iraq durante las décadas anteriores a cambio de mejorar la relación comercial, diplomática y estratégica con los turcos. Contra el silencio y la colaboración de Siria, aumentaron el caudal. En efecto, cuando en 2001 las autoridades de Ankara anunciaron que reducirían el flujo debido a la sequía, los sirios expresaron su comprensión. Posteriormente, en 2004, con ocasión de la firma de un tratado bilateral turco-sirio de libre comercio, el primer ministro de Siria, Nayi Mohammad Al-'Otri, declaró que Recep Tayip Erdogan, su contraparte turco, se había comprometido a aumentar el flujo en la frontera. Erdogan confirmó dichos propósitos en diciembre durante su visita a Damasco. ${ }^{22}$

El más supeditado de los tres ribereños en el nuevo esquema sería Iraq, ya que, como se ha mencionado, quedó excluido hasta de las negociaciones. No obstante, le beneficiaba el aumento del caudal, aunque le perjudicaba su reducción en tiem-

${ }^{21}$ Marwa Daoudy, "Asymmetric Power: Negotiating Water in the Euphrates and Tigris”, International Negotiation, vol. 14, núm. 2, 2009, pp. 359-389.

${ }^{22}$ Bülent Araş, “Turkey between Syria and Israel: Turkey's Rising Soft Power", SETA Policy Brief 15, Ankara, Foundation for Political, Economic and Social Research, mayo de 2008. 
pos de sequía. Entre 2001 y 2003, el gobierno de Saddam Husayn en la práctica había participado en los gestos de discreción hacia Turquía en el tema hídrico. Las sanciones y la inminente invasión estadounidense eran su gran preocupación, así como la restauración y el mantenimiento de todo tipo de infraestructura, dañada por las condiciones impuestas por las severas sanciones que le aplicaba Naciones Unidas desde 1990 a instancias de Washington y Londres. A partir de la ocupación en la primavera de 2003, ni Estados Unidos, la potencia invasora, ni los iraquíes participantes de las instancias políticas estaban en condiciones de negociar con sus vecinos ni de confrontarlos en lo referente a los recursos del Tigris y el Éufrates. Se podría haber pensado que usarían el asunto para presionar a Turquía o a Siria con el propósito de obtener concesiones en otros temas, tal como Saddam Husayn había hecho a mediados de los años setenta. Sin embargo, no fue así, al menos no en una escala apreciable.

Los gobernantes turcos han relanzado el esfuerzo de construcción de presas del GAP, principalmente sobre el Tigris. En parte buscan establecer su hidrohegemonía sobre la población kurda de la propia Turquía. Han puesto en marcha las obras ante el relanzamiento de las actividades guerrilleras del PKK desde 2004, luego de que habían silenciado las armas a raíz del llamado hecho por Öcalan desde prisión en 1999. El asunto se planteaba exactamente en esos términos en la prensa turca y extranjera durante 2008. En efecto, el gobierno turco decidió revivir el GAP, que había dejado relativamente estancado. Los funcionarios argumentaban que era necesario crear empleos y prosperidad en el Sureste de Anatolia con el propósito manifiesto de evitar que la población kurda continuara radicalizándose y apoyando a los rebeldes. ${ }^{23}$ No obstante, las administraciones kurdas de oposición en la región expresaban que la Administración de Obras Hídricas del Estado (DSI, por su nombre en turco) traía obreros de otras partes de Turquía para trabajar en

${ }^{23}$ Yigal Schleifer, "Turkey: Dam Project Fosters Development Strategy Debate”, Eurasianet.org, 4 de junio de 2008; Taha Ozhan, "New Action Plan for Southeastern Turkey", sETA Policy Brief 18, Ankara, Foundation for Political, Economic and Social Research, julio de 2008; Ercan Yavuz, "Gov't to launch Action Plan to take on Terrorism”, Today's Zaman, 10 de septiembre de 2008. 
las presas y que esos empleos no beneficiaban a los kurdos. Muchos kurdos pensaban que entre los objetivos de ampliar las obras estaba el de dificultar la movilidad de los insurgentes y el de reprimir a la población que los apoyaba. ${ }^{24}$ En todo caso, si efectivamente la continuación de las obras del GAP buscaba conquistar el apoyo de la población al grupo rebelde, fue un fracaso.

Con un cambio radical respecto de la estrategia de confrontación seguida durante el siglo anterior, Turquía logró establecer una hidrohegemonía mucho más firme sobre Siria e incluso sobre Iraq. Damasco se vio beneficiado al obtener el apoyo de un importante aliado de Estados Unidos cuando la administración del presidente George W. Bush aspiraba a derrocar al gobierno sirio. Ceder parte importante de sus derechos de agua parecía un costo moderado. Hay que decir, no obstante, que el agua del Tigris y el Éufrates era sólo parte del trueque.

En las precarias condiciones en las que se encontraba Iraq en esos años, a sus nuevos gobernantes no les quedaba mucha alternativa frente a una alianza de los dos ribereños superiores que incluía a un país económica, política y militarmente muy fuerte, Turquía.

En cuanto a los kurdos de Turquía, Ankara intentó continuar su postura de hidrodominación aprovechando los recursos a su alcance en la política interna y los clientes que lograba tener en la región del Sureste, que difícilmente le entorpecían sus proyectos de desarrollo de infraestructura hídrica.

Se puede decir que, en la década de 2000, Ankara logró afianzar su hidrohegemonía en la cuenca por medio de sus negociaciones selectivas. De no entender esto, resultaría inexplicable que la relación hídrica haya sido tan cordial entre los tres ribereños a pesar de que los turcos hayan evitado firmar un tratado definitivo de aguas y, más aún, hayan continuado la construcción de presas sin coordinarse con los países corribereños inferiores.

${ }^{24}$ Conde, "Kurdish Counter-hegemony...", op. cit. 


\section{La hidrohegemonía en el contexto del caos y la guerra en Siria}

Las condiciones para el establecimiento de la hidrohegemonía pueden ser aceptables desde el punto de vista de los gobiernos, pero si no toman en consideración las necesidades de las poblaciones, un mecanismo que resulta hidrohegemónico de un Estado sobre otros puede resultar insoportable para sus poblaciones. Las implicaciones resultan mucho más severas si las condiciones ambientales se agravan y, aún más, si coinciden con condiciones de guerra, caos generalizado y acecho de actores bélicos sin escrúpulos, como el caso existente en Siria entre 2011 y 2016, año, éste, en que se escribían estas líneas.

La construcción de presas continuó en Turquía a pesar de la sequía que se vivió durante esos años en la región. La precipitación ha sido tan pobre que Thomas Freedman no duda en asegurar que se convirtió en un factor de peso en el estallido y la extensión de la rebelión popular siria en 2011. ${ }^{25}$ Sin embargo, la importancia del factor hídrico se debe también a que se combinó con el almacenamiento de agua tras las presas ubicadas río arriba.

A partir de 2011, las relaciones entre los países ribereños habían dado un nuevo vuelco. Turquía, junto con sus aliados, apoyó a prácticamente todos los rebeldes sirios que combatieran al gobierno de Damasco. En Iraq, Ankara había tomado partido por la principal coalición de oposición desde 2009 y estableció acuerdos bilaterales con el Gobierno Regional del Kurdistán (GRK) del norte de Iraq sin pasar por las autoridades centrales de Bagdad. El GRK, mientras tanto, se estaba convirtiendo en un actor casi estatal que muy probablemente terminará por desarrollar su propia política del agua, aunque por el momento sigue dependiendo de las autoridades centrales en Bagdad en la política hídrica transnacional. ${ }^{26}$

${ }_{25}$ Thomas Freedman, "Without Water, Revolution", The New York Times, 19 de mayo de 2013.

${ }^{26}$ Conclusiones del autor a raíz de la entrevista que condujo con Mohammed Amín Faris, director general de recursos hídricos del Ministerio de irrigación y recursos hídricos del GRK el 12 de junio de 2013. 
Ya avanzada la situación de guerra, en 2014, por ejemplo, algunos medios de prensa empezaron a denunciar una reducción en el flujo del Éufrates hacia Siria, considerando que se trataba de nuevo de una medida de Ankara para ejercer presiones sobre la política interna de Damasco. ${ }^{27}$ Un mes después, las autoridades turcas negaron que estuvieran cortando el flujo del agua con ese propósito y explicaron que "en promedio" habían estado dejando pasar $599 \mathrm{~m}^{3} / \mathrm{s}$ a pesar de los reportes de que 2014 había sido uno de los años más secos desde 1961. ${ }^{28} \mathrm{El}$ comportamiento, turco según la declaración oficial, se conforma al Documento de Implementación firmado con Siria en 2002. Aunque cualquiera de las declaraciones o ambas fueran producto de propaganda, hablan de las sensibilidades y animosidades que implica el tema.

Por otro lado, la organización llamada Estado Islámico, que, como se sabe, logró controlar extensos territorios en Siria e Iraq a lo largo de la cuenca del Éufrates entre 2014, si no es que desde antes, y 2016, se esforzó por controlar presas y pretendió utilizarlas de manera hidrohegemónica. En abril de 2014, ocupó la de Ramadi sobre el Éufrates, en Iraq, y soltó, según informes periodísticos, un bloque de agua que anegó tierras a una distancia de hasta $160 \mathrm{~km}$ río abajo. ${ }^{29}$ Un año después, utilizaron el control sobre el equipo hidráulico para desviar el flujo del río y poder atacar a las fuerzas gubernamentales. ${ }^{30} \mathrm{El}$ control sobre ésta y otras presas le podría permitir cortar el flujo de energía hidroeléctrica a voluntad, y ocasionar con ello una importante interrupción de todo tipo de actividad o entregar agua a la población para ganar liderazgo sobre ésta. ${ }^{31}$ Los artícu-

${ }^{27}$ Véase, por ejemplo, Suhaib Anjarini, "A New Turkish Aggression against Syria: Ankara Suspends Pumping Euphrates' Water”, Al Akbbar English, 30 de mayo de 2014.

${ }^{28}$ Turkish Ministry of Foreign Affairs, Department of Regional and Transboundary Waters, "Press Release Regarding the Amount of Water that Turkey Releases from the Euphrates River", 4 de julio de 2014.

${ }_{29}$ Nadia Massih, "IsIs Militants use Water as Weapon of War in Iraq", Al-Bawaba, 21 de julio de 2014.

${ }^{30}$ Kashmira Gander, "ISIs Militants use Water as Weapon of War in Iraq, by shutting Dam on the Euphrates River", The Independent, 3 de junio de 2015.

${ }^{31}$ Michael Yalqiyian, “'Dā‘ ish' wa-1-Sirāa ‘ala-1-Miyāh fi Sūriya wa-l-'Irāq”, Beirut Center for Middle East Studies, 25 de septiembre de 2015; Massih, "IsIs Militants use Water as Weapon of War in Iraq", op. cit. 
los citados delatan una preocupación del resto de las fuerzas en el terreno por controlar el líquido vital.

La pregunta obligada es qué hará Turquía con sus proyectos del GAP, sean de irrigación o generación hidroeléctrica, ahora que se han deteriorado los términos de su relación con los gobiernos de los dos países ribereños inferiores, aunque temporalmente tenga relaciones relativamente fluidas con el Gobierno Regional del Kurdistán iraquí. También queda abierta la pregunta de qué hará en caso de que en esos países se establezcan gobiernos con los que tenga buenas relaciones o incluso si se subdividen y se crean nuevos Estados. Si las lecciones del pasado dan alguna indicación de lo que puede traer el futuro, lo más probable es que Ankara no abandonará su tradición de emplear el agua como parte de una estrategia hegemónica en las cuencas del Tigris y el Éufrates, sino que las adaptará a las necesidades cambiantes.

\section{Conclusiones}

Las negociaciones por el agua entre Turquía, Siria e Iraq ponen en evidencia la existencia y la importancia de la hidrohegemonía. A tono con el origen gramsciano del término, nos parece pertinente distinguir entre hidrodominación e hidrohegemonía. A partir de la discusión realizada en el capítulo, el primer concepto debe abarcar aquellas políticas de naturaleza impositiva destinadas a controlar los recursos de un río internacional o a utilizar estos recursos para controlar o presionar a los otros países ribereños. El segundo concepto debería utilizarse de una manera más restrictiva de como se ha empleado hasta ahora para referirse a las medidas de naturaleza negociadora que buscan establecer el liderazgo de un país sobre los demás de una cuenca transfronteriza en asuntos relacionados con sus aguas, aprovechando alguna combinación de posición geográfica, poderío militar, ventajas políticas y fuerza económica. Tanto en la hidrohegemonía como en la hidrodominación, el agua puede ser el objetivo último de las medidas o simplemente un medio.

Con todo, es de observar que las políticas hidrohegemónicas y las de hidrodominación suelen ejercerse en una com- 
binación dialéctica. Así, Turquía, el Estado más poderoso de la cuenca del Tigris y el Éufrates, en ocasiones ha combinado su capacidad de negociación con su fuerza económica, diplomática y militar para presionar a los países ribereños inferiores para que acepten un esquema que le sea favorable.

Durante la primera década del siglo XXI, Turquía y Siria lograron un acercamiento importante en lo económico y lo diplomático que les permitió experimentar con un mecanismo flexible de distribución del agua del Tigris y el Éufrates. Turquía se erigía como el país hegemónico en el expediente del agua al igual que en el resto de la relación bilateral. Siria participaba voluntariamente en el esquema en calidad de parte subordinada aceptando que ganaba algo, aunque menos que su vecino. Las autoridades que se fueron estableciendo en Iraq tras la ocupación estadounidense se tornaron en la parte más subordinada de las tres, aunque gozaban de algunas ganancias relativas.

Turquía logró establecer su hidrohegemonía gracias a varios factores. El final de las tensiones de la guerra fría influyó sin duda, así como algunas acciones que contribuyeron a crear confianza por parte de Siria, como la expulsión de Öcalan o su esfuerzo por asociarse a los capitales turcos. El hecho de que el PKK suspendiera unilateralmente durante varios años sus acciones ofensivas también sirvió.

Asimismo, ocurre que los Estados menos poderosos intentan desplegar medidas hidrohegemónicas o incluso de hidrodominación respecto de los que consideran a su vez menos fuertes. En 1975, el gobierno de Iraq amenazó con invadir Siria, pero no Turquía, para que detuviera el llenado de su nueva presa, una medida de hidrodominación. Al final, su poderío militar incuestionable, basado en la riqueza petrolera, le permitió conseguir un acuerdo de aguas relativamente favorable, en lo que podríamos llamar hidrohegemonía.

Por otro lado, es importante subrayar cómo los actores con menos poder suelen desplegar estrategias de contrahegemonía por medio de las cuales buscan demostrar la naturaleza ilegítima de las medidas de hidrodominación e incluso los intentos puramente hidrohegemónicos del actor o los actores más poderosos. Durante décadas, los dirigentes de Siria e Iraq emplearon 
los medios a su alcance para entrelazar temas. Terminaron por establecer alianzas entre ellos para resistir el poder de Turquía a pesar de que sus administraciones se enfrentaban en otros ámbitos, lo que muestra no sólo la importancia del asunto del agua, sino también la ventaja que pueden encontrar los Estados más débiles en desplegar medidas contrahegemónicas.

Es curioso observar que hay actores no estatales que toman en consideración los temas de la hidrohegemonía; se esfuerzan por establecer mecanismos contrahegemónicos en el tema del agua, como los kurdos civiles en Turquía, o incluso por ejercer su propia hidrodominación, como la organización Estado Islámico en Iraq. Así, los municipios gobernados por partidos prokurdos en Turquía utilizan el tema del agua y el derecho humano al recurso para cuestionar la legitimidad del discurso y la práctica del Estado turco. Algo similar sucede con el GRK en Iraq. En sentido de hidrodominación, la organización Estado Islámico ha buscado tomar diques bajo su control y utilizar el flujo del agua para legitimarse ante la población, para castigarla o para facilitar sus acciones bélicas o entorpecer las del enemigo.

Independientemente de cuán buenas hayan llegado a ser las relaciones entre los países de la cuenca durante la primera década de los años 2000 y qué tan respetuosas hayan sido $\mathrm{y}$ sean de los derechos de aguas, el hecho de que no se haya discutido un acuerdo definitivo, como pedían las autoridades sirias e iraquíes durante los años noventa, pone en evidencia la tendencia de los dos Estados más débiles a caer bajo la hidrohegemonía del más fuerte.

Sin embargo, el hecho de que la construcción de presas haya continuado río arriba en Turquía a pesar del agravamiento de las sequías río abajo, muestra que, aun si los Estados más débiles están dispuestos a aceptar la hidrohegemonía de los más fuertes, si no se toman en consideración las necesidades de las poblaciones, el mecanismo que es hidrohegemónico para los Estados se puede convertir en ruina para las poblaciones.

Dirección institucional del autor:

Centro de Estudios de Asia y África

El Colegio de México, A.C. 
Camino al Ajusco 20

Pedregal de Santa Teresa

10740, Ciudad de México

\section{Bibliografía}

Allan, J. Anthony (ed.), Water, Peace and the Middle East: Negotiating Resources in the Jordan Basin, Londres, St. Martin's Press, 1996.

Amery, Hussein y Aaron T. Wolf, "Water, Geography, and Peace in the Middle East: An Introduction", en Hussein Amery y Aaron T. Wolf (eds.), Water in the Middle East: A Geography of Peace, Austin, Texas University Press, 2000, pp. 1-18.

ANJARINI, Suhaib, "A New Turkish Aggression against Syria: Ankara Suspends Pumping Euphrates' Water”, Al Akhbar English, 30 de mayo de 2014. [english.al-akhbar.com/node/19970, consultado el 12 de julio de 2014.]

ARAş, Bülent, “Turkey between Syria and Israel: Turkey’s Rising Soft Power", sETA Policy Brief 15, Ankara, Foundation for Political, Economic and Social Research, mayo de 2008. [www. setav.org/Ups/dosya/7327.pdf, consultado el 11 de agosto de 2010.]

Ayeb, Habib, L'eau au Proche-Orient : la guerre n'aura pas lieu, París, Karthala, 1998.

Conde, Gilberto, "El agua entre Turquía, Siria e Iraq, ¿barómetro de conflictos?", Regions E Cohesion, vol. 4, núm. 2, verano de 2014, pp. 81-100.

Conde, Gilberto, "Kurdish Counter-hegemony in the Tigris and Euphrates Water Basins: Local Impacts Beyond Water Distribution in Turkey", Local Environment: The International Journal of Justice and Sustainability, 2016 (en prensa).

ConDE, Gilberto, "Turquía e Iraq en las cambiantes relaciones internacionales de Siria”, en Luis Mesa Delmonte (coord.), Las relaciones exteriores de Siria, México, El Colegio de México, A.C., 2013, pp. 159-205.

Daoudy, Marwa, "Asymmetric Power: Negotiating Water in the Euphrates and Tigris", International Negotiation, vol. 14, núm. 2, 2009, pp. 359-389. [graduateinstitute.ch/files/live/sites/iheid/ files/shared/summer/IA2009_readings/MD3.pdf, consultado el 16 de agosto de 2016.] 
Freedman, Thomas, "Without Water, Revolution", The New York Times, 19 de mayo de 2013. [nytimes.com/2013/05/19/opinion/ sunday/friedman-without-water-revolution.html?pagewanted $=$ all\&_ $r=0$, consultado el 15 de junio de 2013.]

GANDER, Kashmira, "ISIs Militants use Water as Weapon of War in Iraq, by shutting Dam on the Euphrates River", The Independent, 3 de junio de 2015. [independent.co.uk/news/world/middleeast/isis-use-water-as-a-weapon-in-iraq-by-shutting-dam-onthe-euphrates-river-10295763.html, consultado el 19 de octubre de 2015.]

Gramsci, Antonio, Selections from the Prison Notebooks of Antonio Gramsci, eds. y trads. Quintin Hoare y Geoffrey Nowell-Smith, Nueva York, International Publishers, 1971.

INAN, Yüksel, "Legal Dimensions of International Watercourse (Euphrates and Tigris)", en Ali Ihsan Bağiş (ed.), Water as an Element of Cooperation and Development in the Middle East, Estambul, Ayna-Hacettepe Universitesi-Friedrich Neuman Foundation, 1994, pp. 223-237.

Kerr, Malcolm H., The Arab Cold War: Gamal Abd al-Nasir and his Rivals, 1958-1970, Londres, Oxford University Press, 1971.

KIBAROĞLU, Ayşegül, Building a Regime for the Waters of the EuphratesTigris River Basin, Londres, Kluwer Law International, 2002.

Kolars, John F. y William A. Mitchell, The Euphrates River and the South East Anatolia Development Project, Carbondale-Edwardsville, Southern Illinois University Press, 1991.

KRISHNA, Raj, "International Watercourses: World Bank Experience and Policy", en J. Anthony Allan y Chibli Mallat (eds.), Water in the Middle East: Legal, Political and Commercial Implications, Londres, I. B. Tauris, 1995, pp. 29-54.

Majzoub, Tarek, Les fleuves du Moyen-Orient, París, L'Harmattan, 1994.

MAssiH, Nadia, "ISIs Militants use Water as Weapon of War in Iraq", Al-Barwaba, 21 de julio de 2014. [albawaba.com/news/isis-wateriraq-591811, consultado el 19 octubre de 2015.]

Murakami, Masahiro, Managing Water for Peace in the Middle East: Alternative Strategies, Tokio, United Nations University Press, 1995.

NafF, Thomas y Ruth C. Matson, Water in the Middle East: Conflict or Cooperation?, Boulder, Westview Press, 1984.

Özhan, Taha, "New Action Plan for Southeastern Turkey", sETA Policy Brief 18, Ankara, Foundation for Political, Economic and Social Research, julio de 2008. [arsiv.setav.org/public/ 
HaberDetay.aspx?Dil = tr\&hid $=7365 \&$ q $=$ new-action-plan-forsoutheastern-turkey, consultado el 16 de agosto de 2016].

SCHLEIFER, Yigal, “Turkey: Dam Project Fosters Development Strategy Debate”, Eurasianet.org, 4 de junio de 2008. [eurasianet.org/ departments/insight/articles/eav060408a.shtml, consultado el 4 de junio de 2008.]

Selby, Jan, "The Geopolitics of Water in The Middle East: Fantasies and Realities", Third World Quarterly, vol. 26, núm. 2, junio de 2005, pp. 329-349. [dx.doi.org/10.1080/0143659042000339146.] Shapland, Greg, Rivers of Discord: International Water Disputes in the Middle East, Nueva York, St. Martin's Press, 1997.

Shuval, Hillel I. y Hassan Dwiek (coords.), Water Resources in the Middle East: The Israeli-Palestinian Water Issues: From Conflict to Cooperation, Berlín, Springer, 2007.

Turkish Ministry of Foreign Affairs, Department of Regional and Transboundary Waters, "Press Release Regarding the Amount of Water that Turkey Releases from the Euphrates River", 4 de julio de 2014. [www.mfa.gov.tr/no_-228_-4-july-2014_-pressrelease-regarding-the-amount-of-water-that-turkey-releases-fromthe-euphrates-river.en.mfa, consultado el 12 de julio de 2014.]

Wagner, Wolfgang, Groundwater in the Arab Middle East, Heidelberg, Springer, 2011.

Warner, Jeroen, "Hydro-Hegemonic Politics: A Crossroads on the Euphrates-Tigris?", en Kai Wegerich y Jeroen Warner (eds.), The Politics of Water: A Survey, Londres, Routledge, 2010, pp. 119141.

WARNER, Jeroen, Mark Zeitoun y Naho Mirumachi, "How 'Soft Power' Shapes Transboundary Water Interaction”, GWF Discussion Paper 1323, Canberra, Global Water Forum, junio de 2013. [www.globalwaterforum.org/2013/06/03/6928/, consultado el 14 diciembre de 2013.]

Wolf, Aaron T., "'Hydrostrategic Territory' in the Jordan Basin: Water, War, and Arab-Israeli Peace Negotiations", en Hussein Amery y Aaron T. Wolf (eds.), A Geography of Peace: Water in the Middle East, Austin, Texas University Press, 2000, pp. 63-120.

YALQIYIAN, Michael, “'Dā‘ ish’ wa-l-Sirāa ‘ala-l-Miyāh fi Sūriya wa-l'Irāq”, Beirut Center for Middle East Studies, 25 de septiembre de 2015. [beirutme.com/?p=3187, consultado el 19 de octubre de 2015.]

Yavuz, Ercan, "Gov't to launch Action Plan to take on Terrorism”, Today's Zaman, 10 de septiembre de 2008. [www.todayszaman. 
com/news-152718-govt-to-launch-action-plan-to-take-on-terrorism.html, consultado el 23 de noviembre de 2008.]

Zeitoun, Mark y Jeroen Warner, "Hydro-Hegemony: A Framework for Analysis of Trans-Boundary Water Conflicts", Water Policy, vol. 8, 2006, pp. 435-460.

Zeitoun, Mark, Naho Mirumachi y Jeroen Warner, “Transboundary Water Interaction II: The Influence of 'Soft' Power”, International Environmental Agreements: Politics, Law and Economics, vol. 11, núm. 2, 2011, pp. 159-178. [dx.doi.org/10.1007/s10784010-9134-6].

Zeitoun, Mark, Power and Water in the Middle East: The Hidden Politics of the Palestinian-Israeli Water Conflict, Londres, I. B. Tauris, 2011. 